\title{
Beam instability and microbunching due to coherent synchrotron radiation
}

\author{
G. Stupakov and S. Heifets \\ Stanford Linear Accelerator Center, Stanford University, Stanford, California 94309
}

(Received 7 February 2002; published 21 May 2002)

\begin{abstract}
A relativistic electron beam moving in a circular orbit in free space can radiate coherently if the wavelength of the synchrotron radiation exceeds the length of the bunch. In accelerators coherent synchrotron radiation of the bunch is usually suppressed by the shielding effect of the conducting walls of the vacuum chamber. However an initial density fluctuation with a characteristic length much shorter than the bunch length can radiate coherently. If the radiation reaction force results in the growth of the initial fluctuation, one can expect an instability which leads to microbunching of the beam and an increased coherent radiation at short wavelengths. Such an instability is studied theoretically in this paper.
\end{abstract}

DOI: 10.1103/PhysRevSTAB.5.054402

PACS numbers: $41.75 . J v, 41.75 . \mathrm{Ht}$

A relativistic electron beam moving in a circular orbit in free space can radiate coherently if the wavelength of the synchrotron radiation exceeds the length of the bunch. In accelerators coherent radiation of the bunch is usually suppressed by the screening effect of the conducting walls of the vacuum chamber [1-3]. The screening effect is much less effective for short wavelengths, but if the wavelength is shorter than the length of the bunch (assuming a smooth beam profile), the coherent radiation becomes exponentially small. However, an initial density fluctuation with a characteristic length much shorter than the screening threshold would radiate coherently. If the radiation reaction force is directed so that it results in the growth of the initial fluctuation one can expect an instability that leads to microbunching of the beam and an increased coherent radiation at short wavelengths. Experimentally self-excited coherent microwave radiation has been observed in the National Synchrotron Light Source (NSLS) VUV ring at Brookhaven National Laboratory [4], at the Synchrotron Ultraviolet Radiation Facility at the National Institute of Standards and Technology [5], and at the Advanced Light Source (ALS) at the Lawrence Berkelely Laboratory [6]. Microbunching in numerical simulations of a bunch compressor was reported in Ref. [7] and, more recently, was found in the simulations of the Linac Coherent Light Source bunch compressor [8].

A beam microwave instability induced by the coherent radiation of the density fluctuations is studied theoretically in this paper. Throughout this paper we assume that the characteristic wavelength of the instability is small compared to the bunch length. In this case, the development of the instability is governed by the local value of the beam current and one can simplify consideration neglecting variation of the beam density within the bunch.

Consider a coasting beam of energy $E_{0}$ moving in a circular orbit of radius $R$ in free space. Let us use $(\delta, z)$ variables, where $\delta$ is the relative energy offset of a particle, $\delta=\left(E-E_{0}\right) / E, z$ is the longitudinal coordinate measured relative to the reference particle with the nominal energy, and $s=c t$.
The beam is described by the longitudinal distribution function $\rho(\delta, z, s)$ normalized so that $d N=$ $d z \int \rho(\delta, z, s) d \delta$ gives the number of particles at point $z$ within an infinitesimal interval $d z$. For an instability that is slow compared to the frequency of the betatron oscillations $\omega_{\beta},|\omega| \ll \omega_{\beta}$, one can use a one-dimensional Vlasov equation for the distribution function $\rho$ [9]:

$$
\begin{gathered}
\frac{\partial \rho}{\partial s}-\eta \delta \frac{\partial \rho}{\partial z}- \\
\frac{r_{0}}{\gamma} \frac{\partial \rho}{\partial \delta} \int_{-\infty}^{\infty} d z^{\prime} d \delta^{\prime} W\left(z-z^{\prime}\right) \rho\left(\delta^{\prime}, z^{\prime}, s\right)=0,
\end{gathered}
$$

where $\eta$ is the slip factor, $r_{0}$ is the classical electron radius, and the wake function per unit length of the path $W(z-$ $\left.z^{\prime}\right)$ describes the interaction with the coherent synchrotron radiation (CSR). In this equation we omitted the effects of the incoherent synchrotron radiation damping. Such an approximation is valid if the growth rate of the instability is much faster than the inverse synchrotron radiation damping time.

Neglecting the screening effect of conducting walls we will use the wake function corresponding to the steadystate radiation of an ultrarelativistic particle $(\gamma \gg 1)$ in a long magnet $[10,11]$,

$$
W(z)=\frac{2}{\left(3 R^{2}\right)^{1 / 3}} \frac{\partial}{\partial z} z^{-1 / 3} \text { for } z>0,
$$

and $W(z)=0$ for $z \leq 0$. Note that unlike the traditional wake the radiation wakefield is localized in front of the moving charge. Equation (2) neglects transient effects occurring at the entrance to and exit from the magnet.

We represent the distribution function $\rho$ as a sum of the equilibrium distribution function $\rho_{0}$ and a perturbation $\rho_{1}$,

$$
\rho=\rho_{0}(\delta)+\rho_{1}(\delta, z, s),
$$

with $\rho_{1} \ll \rho_{0}$. Note that the equilibrium beam density (number of particles per unit length) $n_{b}$ is equal to $n_{b}=$ $\int \rho_{0}(\delta) d \delta$, and the density perturbation $n_{1}$ is given by $n_{1}(z, s)=\int \rho_{1}(\delta, z, s) d \delta$. Linearizing Eq. (1) and assuming that 


$$
\rho_{1}=\hat{\rho}_{1} e^{-i \omega s / c+i k z},
$$

where $k$ is the wave number of the perturbation, we find

$$
(\omega+c k \eta \delta) \hat{\rho}_{1}=i \frac{r_{0} c}{\gamma} \frac{\partial \rho_{0}}{\partial \delta} Z(k) \int d \delta \hat{\rho}_{1}(\delta),
$$

where

$$
\begin{aligned}
Z(k) & =\int_{0}^{\infty} d \zeta W(\zeta) e^{-i k \zeta} \\
& =\frac{2 i k^{1 / 3}}{3^{1 / 3} R^{2 / 3}} \int_{0}^{\infty} d \xi \xi^{-1 / 3} e^{-i \xi}=-i A \frac{k^{1 / 3}}{R^{2 / 3}}
\end{aligned}
$$

The complex factor $A$ is

$$
A=3^{-1 / 3} \Gamma\left(\frac{2}{3}\right)(\sqrt{3} i-1)=1.63 i-0.94,
$$

where $\Gamma$ is the complete gamma function.

The nontrivial solution of Eq. (5)

$$
\hat{\rho}_{1}=\frac{i c r_{0} Z(k)}{\gamma(\omega+c k \eta \delta)} \frac{d \rho_{0}}{d \delta} \hat{n}_{1}
$$

exists if $\omega$ satisfies the dispersion relation

$$
1=\frac{i r_{0} c Z(k)}{\gamma} \int \frac{d \delta\left(d \rho_{0} / d \delta\right)}{\omega+c k \eta \delta},
$$

where $\hat{n}_{1}$ in Eq. (8) is the amplitude of the density perturbation, $\hat{n}_{1}=\int d \delta \hat{\rho}_{1}$. For the Gaussian distribution function, $\rho_{0}=n_{b}(2 \pi)^{-1 / 2} \exp \left(-\delta^{2} / 2 \delta_{0}^{2}\right)$, where $\delta_{0}$ is the rms relative energy spread, we can write Eq. (9) in the following form:

$$
\frac{(k R)^{2 / 3}}{\Lambda}=-\frac{A}{\sqrt{2 \pi}} \int_{-\infty}^{\infty} \frac{d p p e^{-p^{2} / 2}}{\Omega \pm p},
$$

where

$$
\Lambda=\frac{n_{b} r_{0}}{|\eta| \gamma \delta_{0}^{2}},
$$

$\Omega=\omega / c k|\eta| \delta_{0}$, and the upper (lower) sign in Eq. (10) refers to the case of positive (negative) $\eta$. As always in stability theory, the integral on the right-hand side of Eq. (9) defines the dispersion function in the upper half plane of the complex variable $\omega$; the values of this function for $\operatorname{Im} \omega<0$ are obtained by analytic continuation of the integral into the lower half plane.

The plot of the normalized frequency $\omega$ as a function of the wave number $k$ obtained by the numerical solution of Eq. (10) for the positive value of $\eta$ is shown in Fig. 1.

The imaginary part of the frequency is positive, and the beam is unstable, for

$$
k R<2.0 \Lambda^{3 / 2} .
$$

The maximum growth rate is reached at $k=0.68 \Lambda^{3 / 2} / R$ and is equal to $(\operatorname{Im} \omega)_{\max }=0.43 \Lambda^{3 / 2} c|\eta| \delta_{0} / R$. Note that the condition for the instability is easier to satisfy for a beam with a small energy spread, since $\Lambda \propto 1 / \delta_{0}^{2}$. This condition does not take into account the finite bunch length,

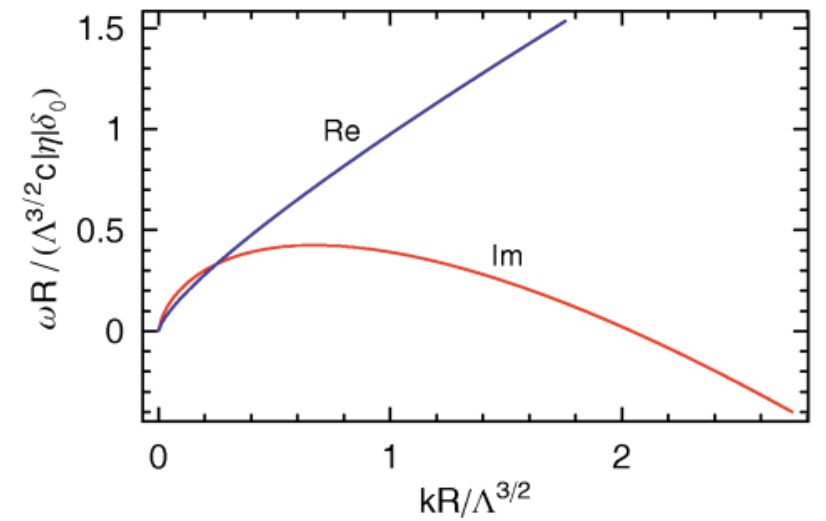

FIG. 1. (Color) The imaginary (Im) and real (Re) parts of the frequency $\omega$ as functions of $k R / \Lambda^{3 / 2}$, for a positive value of $\eta$. For negative values of $k$, the frequency can be found from the relation $\omega(-k)=-\omega^{*}(k)$ which follows from Eq. (9).

the screening effect of the vacuum chamber walls, and the beam emittance (see below).

The numerical solution for a negative value of $\eta$ gives a higher threshold for the instability $k R<0.92 \Lambda^{3 / 2}$ with the maximum growth rate $(\operatorname{Im} \omega)_{\max }=0.16 \Lambda^{3 / 2} c|\eta| \delta_{0} / R$ at $k=0.31 \Lambda^{3 / 2} / R$.

From the dispersion relation Eq. (9) it is easy to obtain an explicit expression for the frequency of the instability in the limit of a cold beam, when $k \ll \Lambda^{3 / 2} / R$,

$$
\omega=c\left(\frac{A r_{0} k^{4 / 3} n_{b} \eta}{\gamma R^{2 / 3}}\right)^{1 / 2}
$$

For a bunched beam of length $\sigma_{z}$ with $N$ particles in the bunch, our results obtained in the coasting-beam approximation can be applied if the reduced wavelength of the modulation $1 / k$ is much smaller than the bunch length, $k \sigma_{z} \gg 1$. In this case the instability is controlled by the local value of the linear particle density $n_{b}$, with the maximum value of $n_{b}$, for a Gaussian bunch, equal to $N / \sqrt{2 \pi} \sigma_{z}$. Since $k$ is limited from above by the requirement (12), the microbunching instability can develop if the bunch length is large enough

$$
\sigma_{z} \geq 0.5 R \Lambda^{-3 / 2} \text {. }
$$

Another limitation to the theory is introduced by a finite aperture $b$ of the beam pipe. Assuming a pipe with a perfect conductivity, the CSR is suppressed due to the shielding effect at low harmonics with wave numbers $k$ such that $k R \lessgtr(\pi R / 2 b)^{3 / 2}$ [12]. Hence the instability can only develop for such values of $k$ that $2.0 \Lambda^{3 / 2}>k R \geqslant$ $(\pi R / 2 b)^{3 / 2}$. The requirement that the lower limit for the parameter $k R$ is smaller than the upper one gives another necessary condition for the instability:

$$
\frac{R}{b} \lesssim \Lambda \text {. }
$$

If this inequality is not satisfied, finite energy spread in the beam suppresses the small-scale instability where the effect of the screening is not essential. Note that for 
$\Lambda \sim R / b$ the condition (14) is just opposite to the condition of a bunch radiating as a whole.

Although we neglected the transverse emittance of the beam in our consideration, its effect can be easily estimated [13]. A betatron oscillation with an amplitude $\Delta x$ inside a bending magnet, through the equation $d z / d s=x / R$, couples to the longitudinal motion with the amplitude $\Delta z \sim c \Delta x / \omega_{\beta} R$. If this amplitude becomes comparable to the reduced wavelength of the instability $k^{-1}$, interaction of the particle with the wake weakens. Hence our results are valid for $k c \sigma_{x} / \omega_{\beta} R \ll 1$ where $\sigma_{x}$ is the rms beam size in the horizontal plane. A more detailed treatment of the effect of the horizontal emittance of the beam on the instability can be found in Ref. [14].

The above equations were derived for a circular orbit of the beam when $R=$ const. If this is not the case, one has to average the wakefield over the beam orbit which results in an additional factor $R /\langle R\rangle$ in Eq. (2), where $R$ is the bending radius of the magnets, and $\langle R\rangle=C / 2 \pi$ is the average radius of the beam orbit of circumference $C$. With this modification, instead of Eq. (11), one should use the following expression for the parameter $\Lambda$ :

$$
\Lambda=\frac{n_{b} r_{0} R}{|\eta| \gamma \delta_{0}^{2}\langle R\rangle},
$$

in conditions Eqs. (14) and (15).

The CSR wake, Eq. (2), is derived assuming that all particles in the beam move along the same circular orbit of radius $R$ with the speed of light. During instability, due to the energy perturbation caused by the wake, the projected velocity is $v=c(1-\eta \delta)$ [15]. To find the condition when the correction to the wake due to this effect can be neglected, we will require the wake formation time be much smaller than the oscillation period of the instability:

$$
t_{\text {form }} \sim \frac{R}{c} \frac{1}{(k R)^{1 / 3}} \ll \frac{1}{\omega} .
$$

Using for the characteristic wave number and frequency of the instability $k R \sim \Lambda^{3 / 2}$ and $\omega \sim \Lambda^{3 / 2} c|\eta| \delta_{0} / R$ yields the condition of applicability of the theory

$$
\frac{n_{0} r_{0}}{\delta_{0} \gamma} \ll 1
$$

Finally, one has to take into account the effect of the incoherent synchrotron damping in the ring, which was neglected in Eq. (1). As a crude estimate of this effect, we can assume that the beam is unstable if the imaginary part of the frequency calculated above exceeds the synchrotron damping time $\gamma_{d}$. The effective growth rate of the instability in this case will be given by the difference $\operatorname{Im} \omega-\gamma_{d}$ (for a detailed treatment of this issue, see Ref. [16]).

We estimated conditions for the instability in the Low Energy Ring (LER) of the PEP-II accelerator at SLAC, the ALS at the Berkeley National Laboratory, and the VUV ring at the National Synchrotron Light Source at BNL. For the ALS, we use beam parameters for the regime in which bursts of infrared radiation were observed [6].
The machine parameters and the calculated values of $\Lambda, R / 2 \Lambda^{3 / 2}$, and the ratio $R / b$ are presented in Table I. For LER the parameter $\Lambda$ is smaller than the ratio $R / b$ so that the condition (15) is not satisfied. For ALS and the VUV ring the condition (15) is satisfied. The parameter $R / 2 \Lambda^{3 / 2}$, which should be compared with the bunch length [see Eq. (14)], is larger than $\sigma_{z}$ for ALS and VUV rings. Hence, according to the theory, the CSR instability is possible in these machines, which may explain observations of bursts of coherent radiation in the experiment $[4,6]$.

The above results are valid for the initial linear regime of the instability, when the amplitude of the perturbation is small. In the unstable situation, the growth of an initial perturbation will eventually evolve into a nonlinear regime where the linear model breaks down. One can expect a saturation of the instability at some level in the nonlinear regime. We will estimate here the amplitude of the instability at the saturation using simple order of magnitude arguments; a more detailed study of the nonlinear regime is published elsewhere [17].

Let us assume that the perturbation of the beam density can be written as $\hat{n}_{1} e^{-i \omega s / c+i k z}$. The wake that is generated by this perturbation is

$$
\begin{aligned}
w_{1} & =\int_{-\infty}^{\infty} d z^{\prime} W\left(z-z^{\prime}\right) n_{1}\left(z^{\prime}, s\right) \\
& =i A \hat{n}_{1} \frac{k^{1 / 3}}{R^{2 / 3}} e^{-i \omega s / c+i k z}
\end{aligned}
$$

The particles in the beam move under the influence of this wake according to the equation of motion $d \delta / d s=$ $-r_{0} / \gamma w_{1}$. Integrating this equation, one finds

$$
\delta=\frac{A \hat{n}_{1} r_{0} c k^{1 / 3}}{\gamma R^{2 / 3} \omega} e^{-i \omega s / c+i k z} .
$$

When the amplitude of the oscillations becomes comparable to the width of the distribution function, $\delta \sim \delta_{0}$, the linear theory breaks down, and one can expect a saturation of the instability. This gives an order of magnitude estimate for the maximum density perturbation

TABLE I. Beam and instability parameters for LER, ALS, and VUV rings.

\begin{tabular}{cccc}
\hline \hline Accelerator & LER PEP-II & ALS & VUV NSLS \\
\hline$E(\mathrm{GeV})$ & 3.1 & 1.5 & 0.81 \\
$\eta$ & $1.31 \times 10^{-3}$ & $1.41 \times 10^{-3}$ & $2.35 \times 10^{-2}$ \\
$\delta_{0}$ & $8.1 \times 10^{-4}$ & $7.1 \times 10^{-4}$ & $5.0 \times 10^{-4}$ \\
$\langle R\rangle(\mathrm{m})$ & 350 & 31.3 & 8.11 \\
$R(\mathrm{~m})$ & 13.7 & 4 & 1.91 \\
$b(\mathrm{~cm})$ & 2 & 1 & 2.1 \\
$I_{b}(\mathrm{~mA})$ & 2 & 30 & 400 \\
$\sigma_{z}(\mathrm{~cm})$ & 1 & 0.7 & 4.7 \\
$\Lambda$ & 7 & $1.2 \times 10^{3}$ & 250 \\
$R / b$ & 550 & 400 & 90 \\
$R / 2 \Lambda^{3 / 2}(\mathrm{~cm})$ & 1.0 & $4.7 \times 10^{-5}$ & 0.025 \\
\hline \hline
\end{tabular}




$$
\frac{\hat{n}_{1}}{n_{b}} \sim \frac{\gamma \delta_{0}^{2} \eta(k R)^{2 / 3}}{r_{0} n_{b}}=\frac{(k R)^{2 / 3}}{\Lambda}
$$

where we have used the relation $\omega \sim c k \eta \delta_{0}$. Of course, this formula is valid if the density perturbation is smaller than the equilibrium beam density, $\hat{n}_{1} \lesssim n_{b}$, which is satisfied above the threshold due to Eq. (12).

Note that for very small values of $\delta_{0}$ (and hence large $\Lambda$ ) the relative density perturbation below which the linear theory is applicable becomes very small, $\hat{n}_{1} / n_{b} \ll 1$.

It is important to emphasize here that the wake Eq. (2) used in this paper is not applicable for very short wavelengths. Indeed, this wake was derived for a bunch that is infinitely thin in the transverse direction and assumes that all particles in the cross section of the bunch radiate coherently. However, the transverse coherence length $l_{\perp} \sim k^{-2 / 3} R^{1 / 3}$ decreases with the wavelength and for very large values of $k$ becomes smaller than the transverse dimension of the beam. For such a wavelength, one has to use a wake that takes into account the transverse dimension of the beam.

In summary, we showed that the coherent synchrotron radiation can produce a bunch modulation provided that the linear bunch density is above the threshold given by the criterion Eq. (12). The instability occurs in a range of wave numbers $k$. Modes with small wavelengths are stabilized by the energy spread in the beam, and the large wavelengths can be stabilized either by the screening effect of the vacuum chamber or by the finite length of the bunch. It is worthwhile to emphasize that the threshold depends on the rms energy spread $\delta_{0}$ and that cooling of a bunch with given bunch population makes the bunch more susceptible to the instability.

We thank R. Warnock for useful discussions. This work was supported by the Department of Energy Contract No. DE-AC03-76SF00515.

[1] J. Schwinger (unpublished).

[2] L. Schiff, Rev. Sci. Instrum. 17, 6 (1946).

[3] R. L. Warnock and P. Morton, Part. Accel. 25, 113 (1990).

[4] G. L. Carr et al., Nucl. Instrum. Methods Phys. Res., Sect. A 463, 387 (2001).

[5] U. Arp et al., Phys. Rev. ST Accel. Beams 4, 054401 (2001).

[6] J. Byrd (private communication).

[7] M. Borland, Phys. Rev. ST Accel. Beams 4, 074201 (2001).

[8] P. Emma and M. Borland (private communication).

[9] Alexander W. Chao, Physics of Collective Beam Instabilities in High Energy Accelerators (Wiley, New York, 1993).

[10] J. B. Murphy, S. Krinsky, and R. L. Gluckstern, Part. Accel. 57, 9 (1997).

[11] Y.S. Derbenev et al., DESY Report No. TESLA-FEL 95-05, 1995.

[12] R. L. Warnock, SLAC Report No. SLAC-PUB-5375, 1990.

[13] E. L. Saldin, E. A. Schneidmiller, and M. V. Yurkov, DESY Report No. TESLA-FEL 2002-2, 2002.

[14] S. Heifets, S. Krinsky, and G. Stupakov (to be published).

[15] For negative values of $\delta$, formally $v>c$. This however does not violate the special theory of relativity, because $v$ is not the velocity of a material object.

[16] S. Heifets and G. Stupakov, SLAC Report No. SLACPUB-8803, 2001.

[17] S. Heifets and G. Stupakov, SLAC Report No. SLACPUB-8758, 2001. 